\title{
Performance evaluation of video streaming on LTE with coexistence of Wi-Fi signal
}

\author{
Y. J. I. A. Yaqoob, W. L. Pang, S. K. Wong, K. Y. Chan \\ Faculty of Engineering, Multimedia University, Cyberjaya, Malaysia
}

\begin{tabular}{ll}
\hline \hline Article Info & ABSTRACT \\
\cline { 2 - 3 } Article history: & $\begin{array}{l}\text { The continuous growth in mobile data traffic and limited license wireless } \\
\text { spectrum have led to dramatically increase the demand of the radio spectrum. }\end{array}$ \\
Received Jan 2, 2019 & $\begin{array}{l}\text { It is widespread the concern about the coexistence of long term evolution } \\
\text { (LTE) and Wi-Fi in the unlicensed band. There are several techniques have } \\
\text { Revised Mar 10, } 2019\end{array}$ \\
Accepted Mar 29,2019 & $\begin{array}{l}\text { band, but these works are targeted on the impact of the LTE to the Wi-Fi } \\
\text { network performance. An experiment is carried out in this work to evaluate }\end{array}$ \\
the impact of Wi-Fi signal on the video streaming in the LTE network. The \\
Keywords:
\end{tabular}$\quad \begin{aligned} & \text { experimental test comprised of the national instrument (NI) universal } \\
& \text { software radio peripheral (USRP) 2953R that is controlled by the LabVIEW } \\
& \text { Communication LTE application framework. Extensive experiments are } \\
& \text { LabVIEW }\end{aligned} \quad \begin{aligned} & \text { carried out under two scenarios, i.e. (1) Coexistence of LTE and Wi-Fi } \\
& \text { signal, (2) LTE signal only. Performance evaluations are carried out with } \\
& \text { different Modulation and coding schemes (MCS) values and different mode } \\
& \text { NI USRP } \\
& \text { of operations, i.e. frequency division duplex (FDD) and time division duplex } \\
& \text { (TDD) mode. The results illustrated that the interference from Wi-Fi signal } \\
& \text { caused the performance degradation of the LTE network in throughput and } \\
& \text { the power received by user equipment (UE). }\end{aligned}$

Copyright @ 2019 Institute of Advanced Engineering and Science. All rights reserved.

\section{Corresponding Author:}

W. L. Pang,

Faculty of Engineering,

Multimedia University,

63100, Cyberjaya, Selangor, Malaysia.

Email:wlpang@mmu.edu.my

\section{INTRODUCTION}

Long term evolution (LTE) represents an emerging technology as a new standard in mobile communication that provides QoS for multimedia services. However, it is not specified in 3GPP the scheduling algorithm that supports real-time and best effort application services [1]. For this reason, there is a vast number of researches have been done that proposed a variety of algorithms to support data transmission over the LTE network. LTE network offers scalable bandwidth from 1.25 to $20 \mathrm{MHz}$ in release 8 [2], peak data rates of $300 \mathrm{Mbps}$ and $75 \mathrm{Mbps}$ for downlink and uplink respectively, and enhance support for an end to end QoS [3]. Furthermore, in release 9, LTE offers the new features such as multimedia broadcast services, dual-layer beamforming, and location-based services [4-5]. LTE employs orthogonal frequency division multiplexing (OFDM) and single-carrier frequency division multiple access schemes for downlink and uplink multiple access schemes respectively [6]. Two of the significant building blocks in LTE-A is the bandwidth extension in the form of the carrier aggregation (CA) as well as heterogeneous network (HetNets) performance [7]. CA is employed to improve the capacity of the cell [8]. In order to achieve up to $1 \mathrm{~Gb} / \mathrm{s}$ peak data rate in future International Mobile Telecommunications-Advanced mobile systems, CA technology is introduced by the 3GPP to support very-high-data-rate transmissions over wide frequency bandwidths in its new LTE-Advanced standards [9-10]. At present, the mobile data traffic has rapidly grown and it has led to the scarcity of radio spectrum In a way that it has been estimated that wireless data traffic in the year of 
2020 will reach 1000 times compared to 2010 [11]. A vast number of researches were carried out on the LTE unlicensed band since the demand for radio spectrum has increased over the years. To cope with the scarcity of the radio spectrum, there is an extension of the LTE network that operates on both licensed and unlicensed $5 \mathrm{GHz}$ spectrum, which widely used by Wi-Fi access points. Several techniques are proposed to allow the fairly coexist of the LTE and Wi-Fi signals on the shared spectrum. One of the proposed solutions for the spectrum scarcity problem is the cognitive radio (CR) technique. Spectrum sensing (SS) is one of the CR technique that used to detect the spectrum hole of the primary user. There are several other SS approaches proposed in LTE-A system such as Auto-Correlation based Advanced Energy spectrum sensing algorithm [12], Spectrum sensing based on time domain energy detection method [13], and Pilot Induced Cyclostationarity and Gaussian Maximum-Likelihood spectrum sensing algorithms [14]. On the other hand, there are researches have been done to enable LTE and Wi-Fi systems to exist in an unlicensed band. The author in [15] proposed an algorithm to enhance the performance of the uplink in a unified technology framework, named licensed-assisted access (LAA). LAA is a carrier aggregation to transmit data on the unlicensed spectrum in LTE-A, which is required to perform the listen-before-talk checking. It significantly decreases the resource utilization [15]. The author in [16] proposed the Q-learning algorithm which dynamically allocates the resources to the users through the new frame structure. In [17], the authors reviewed the existing techniques that enable the LTE and Wi-Fi signals to fairly coexist in the unlicensed band. So far most of the researches are done on how the LTE network affects the Wi-Fi network operation. The overall results showed that when LTE and Wi-Fi signals are operating on the same shared spectrum, Wi-Fi suffers more compare to LTE. Since LTE is designed to operate in a licensed band and it occupies the spectrum continuously. The Wi-Fi MAC protocol is designed based on the carrier-sense multiple access with collision avoidance and the transmission is time-limited [18]. Wi-Fi network was refrained to transmit data when the channel is busy [19]. Extensive works are carried out in this paper to evaluate the LTE network performance with a Wi-Fi signal coexisted using USRP. Performance evaluations are carried out with different Modulation and Coding Schemes (MCS) values and different mode of operations, i.e. frequency division duplex (FDD) and time division duplex (TDD) mode for the LTE network.

The remaining of this work is as follows, i.e. section 2 discussed the research method, section 3 presented the results analysis, and a conclusion is drawn in section 4.

\section{EXPERIMENTAL SETUP}

In this work, a USRP RIO 2563R is programmed as LTE Evolved Node B (eNodeB) and UE as shown in Figure 1. NI Communication Suite 2.0 software is used to program the USRP. An IEEE 802.11ac wireless access point (AP) that operate in $2.4 \mathrm{GHz}$ is placed next to the USRP. There are several APs and radio frequencies in the background environment, which are insignificant compared to this AP after analyzed by the spectrum analyzer. Two experiments are carried out to evaluate the LTE network performance, i.e. (1) When LTE signal coexists with $2.4 \mathrm{GHz}$ Wi-Fi signal as shown in Figure 1. (2) Replaced the LTE antenna with cable as shown in Figure 2 to evaluate the channel under ideal condition. Two different operation modes, i.e. FDD and TDD mode are evaluated under different MCS values. Since the frame structure of FDD and TDD are different and the output of FDD and TDD are also different. For FDD, the uplink and downlink transmissions operate on the separated carrier frequency. However, in TDD operation mode, there is only a single carrier frequency, uplink and downlink transmissions are separated in the time domain within a cell base. Figure 3 depicts the FDD and TDD frame structure for the uplink and downlink transmissions [20].

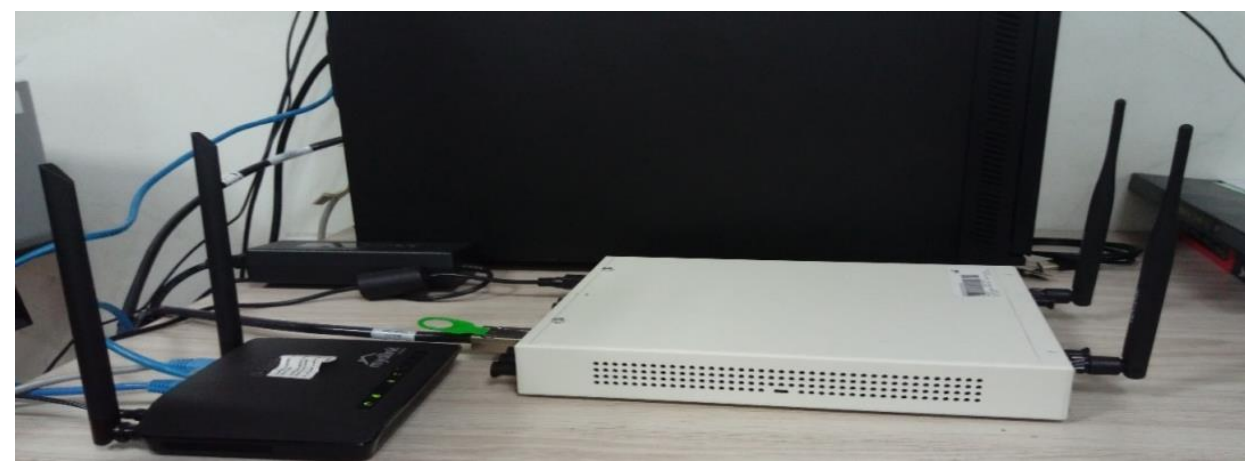

Figure 1. Experimental testbed (USRP and Wi-Fi) 


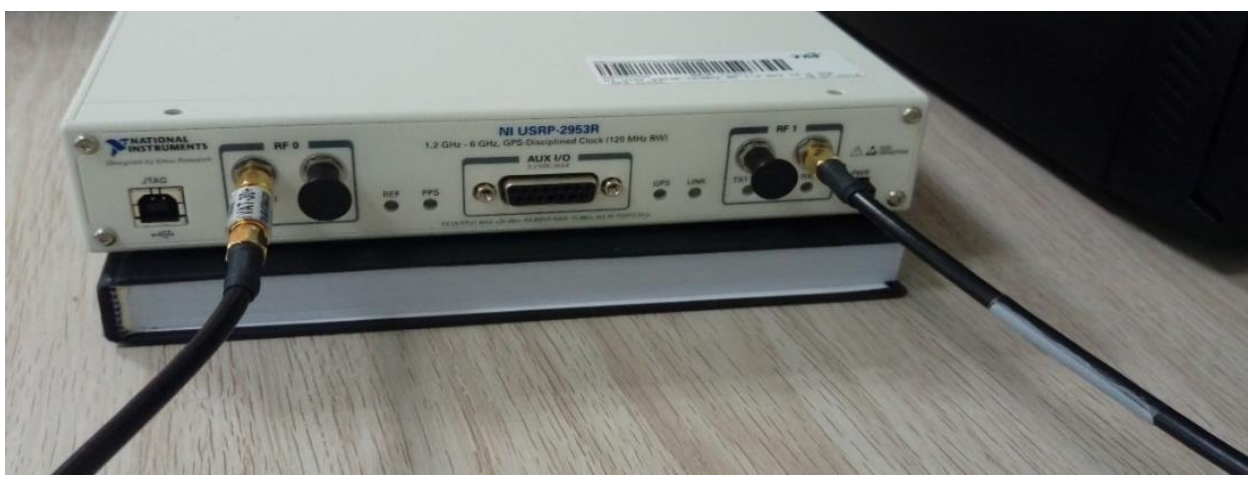

Figure 2. The wire is used to connect the transmitter and receiver for ideal case test

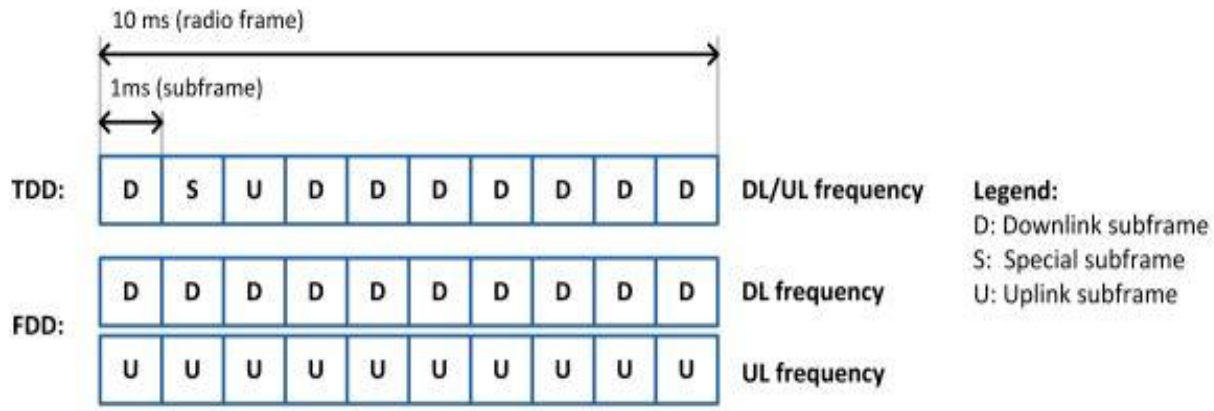
01
23
56
$\begin{array}{lll}6 & 7 & 8\end{array}$
Subframe index

Figure 3. Uplink and downlink frame structure in FDD and TDD [21]

\section{RESULTS AND ANALYSIS}

Extensive experimental tests are carried out to evaluate the LTE network performance. The performance metrics evaluated in this work are the throughput and power received by UE. The MCS values evaluated in this work and their code rates are shown in Table 1. There are 9 MCS values evaluated in this work. The modulation and coding scheme is a 5 bit number, from which UE can look up the modulation scheme, i.e. Quaternary Phase Shift Keying (QPSK), (16-QAM), Quadrature Amplitude Modulation or (64-QAM) that the Physical Downlink Shared Channel (PDSCH) will use and the number of bits in the transport block [22]. In order to optimize the LTE network capacity, UE sends the Channel Quality Indicator (CQI) report to the eNodeB regarding the overall channel condition. A proper modulation scheme will be selected according to the channel condition. If the channel condition is favorable, higher-order MCS with a higher bit rate such as 64 QAM will be used, otherwise, lower-order MCS will be used [23]. There are 28 MCS values in total are available in the NI Communication Suite software under three different modulation schemes (QPSK, 16-QAM and 64-QAM) [24].

Table 1. List of scenarios for experiment set 1 and 2

\begin{tabular}{cccc}
\hline Number & MCS & Coding Rate & Label \\
\hline 1 & MCS 0 & 0.12 & MCS0 \\
2 & MCS 5 & 0.37 & MCS5 \\
3 & MCS 9 & 0.66 & MCS9 \\
4 & MCS 10 & 0.33 & MCS10 \\
5 & MCS 13 & 0.48 & MCS13 \\
6 & MCS 16 & 0.64 & MCS16 \\
7 & MCS 17 & 0.43 & MCS17 \\
8 & MCS 23 & 0.70 & MCS23 \\
9 & MCS 28 & 0.93 & MCS28 \\
\hline
\end{tabular}


As shown in Figure 4 there is a throughput degradation observed from $2.35 \mathrm{GHz}$ to $2.47 \mathrm{GHz}$. The throughput degradation is greater for larger MCS value. MCS 28 (64QAM) with a code rate of 0.93 shown the highest throughput degradation. On the other hand, the throughput for MCS 0 and MCS 5 have zero degradation for the whole spectrum. Lower MCS is more robust to the channel variations since it tolerates better on signal-to-interference-plus-noise ratio (SINR) variation in terms of Block Error Rate (BLER) [25].

Figure 5 illustrates the video throughput for TDD operation mode with the Wi-Fi signal coexist. As shown in Figure 5, it has lower throughput degradation in term of spectrum range, which is between 2.38 to $2.44 \mathrm{GHz}$ that is much lower compared to FDD. In the TDD mode of operation, the Wi-Fi signal has a lower effect on the throughput as the graph shows it has lower degradation compared to the FDD mode of operation. Figure 6 and Figure 7 show the video throughput without Wi-Fi signals. As shown in Figure 2, cable is used to replace the antennas. There is zero degradation for both operation modes under different MCS values. This is shown that the interference from the Wi-Fi signal can significantly decrease the LTE network performance.

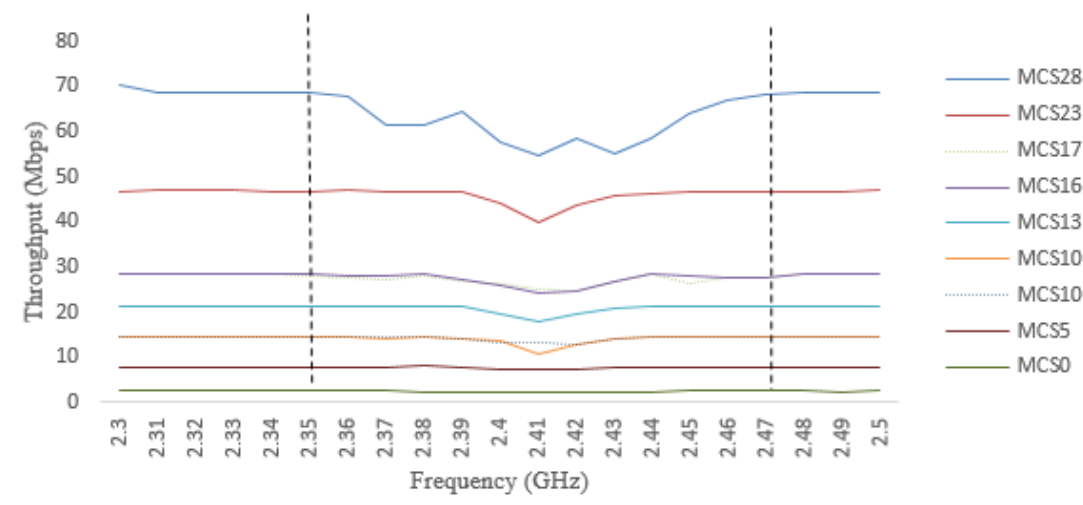

Figure 4. Throughput for FDD operation mode with $2.4 \mathrm{GHz}$ Wi-Fi signal coexist

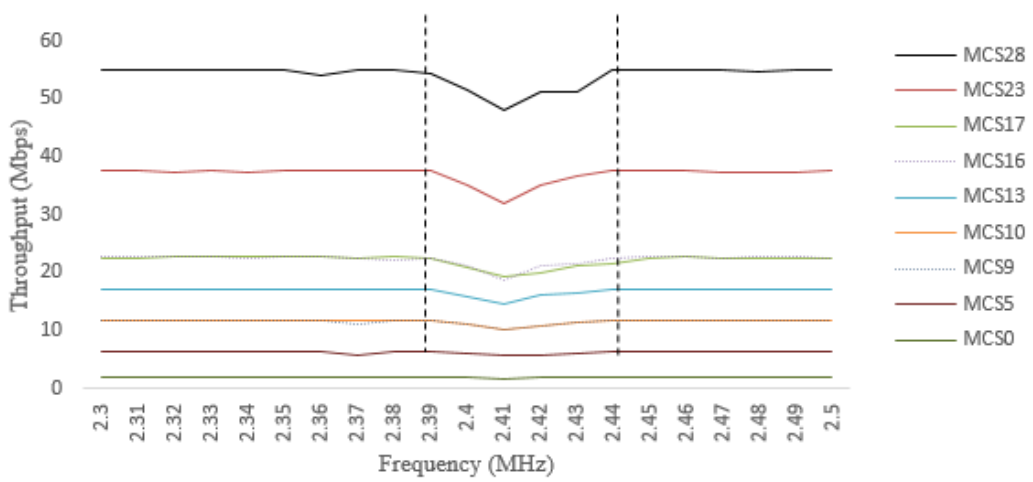

Figure 5. Throughput for TDD operation mode with $2.4 \mathrm{GHz}$ Wi-Fi signal coexist

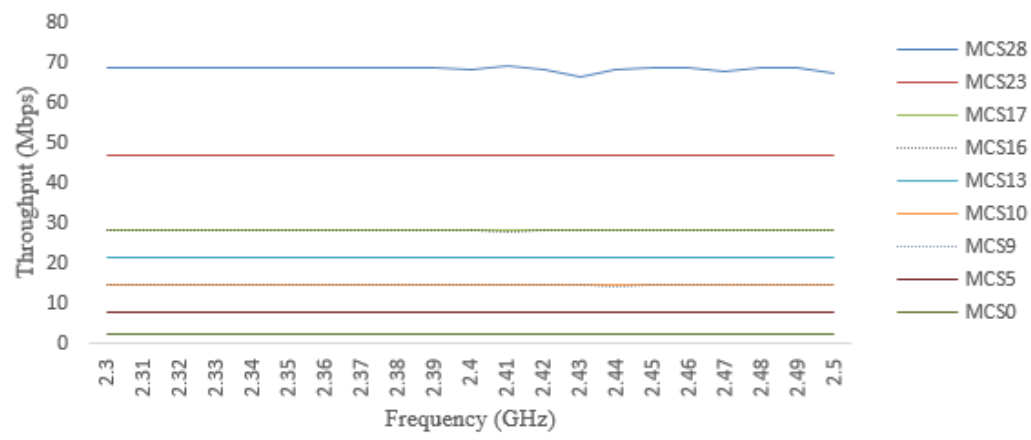

Figure 6. Throughput for FDD operation mode without Wi-Fi signal 


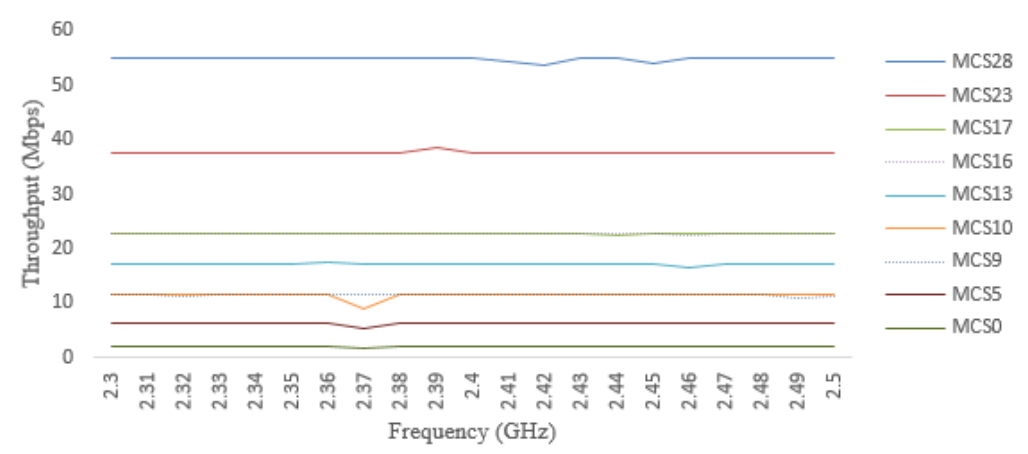

Figure 7. Throughput for TDD operation mode without Wi-Fi signal

The power received by UE for FDD and TDD mode are shown in Figure 8 and Figure 9. As shown in Figure 8, at $2.4 \mathrm{GHz}$ the power received by UE for some MCS values are increased and the MCS values are summarized in Table 2. Based on equation 1; it means that at $2.4 \mathrm{GHz}$, the UE received higher power. This is justifiable since there is an interference between the LTE with Wi-Fi signal. The eNodeB propagates the RF signal with higher power due to the interference from the Wi-Fi signal. Comparing FDD and TDD mode, TDD operation mode has experienced less power fluctuation, since it has less effect from the Wi-Fi signal.

$$
N_{d B}=10 \log \left(\frac{P_{2}}{P_{1}}\right)
$$

Table 2. MCS (modulation and coding scheme)

\begin{tabular}{ccc}
\hline MCS & Modulation & Coding Rate \\
\hline MCS 9 & QPSK & 0.66 \\
MCS 10 & 16QAM & 0.33 \\
MCS 13 & 16QAM & 0.48 \\
MCS 16 & 16QAM & 0.64 \\
\hline
\end{tabular}

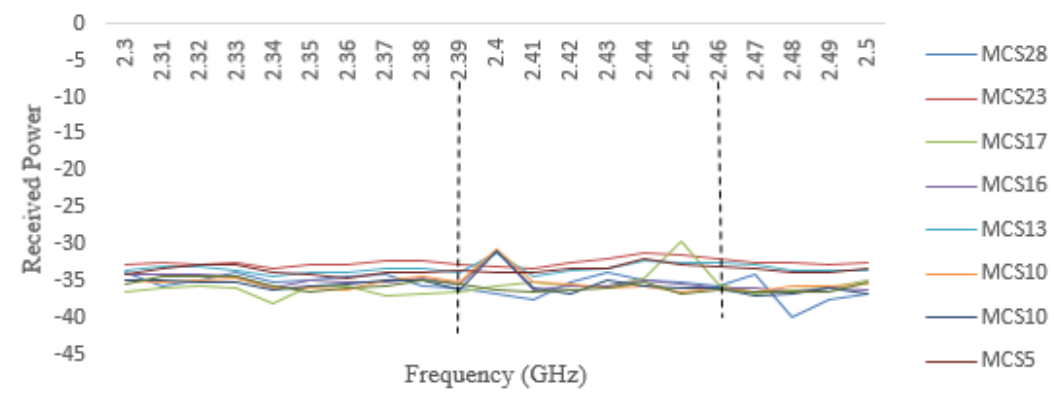

Figure 8. Power received by UE for FDD operation mode with $2.4 \mathrm{GHz}$ Wi-Fi signal coexist

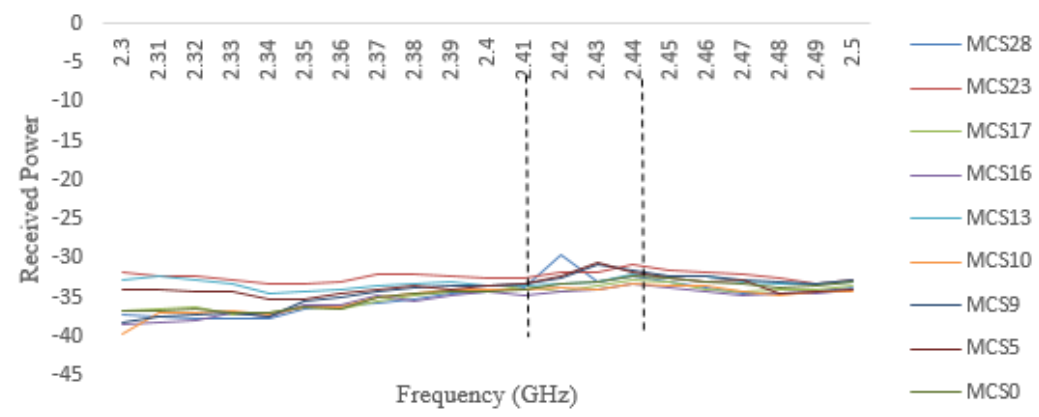

Figure 9. Power received by UE for TDD operation mode with $2.4 \mathrm{GHz}$ Wi-Fi signal coexist 
Figure 10 and Figure 11 show the power received by UE for FDD and TDD modes without Wi-Fi signal (ideal channel condition). As shown in Figure 10 and Figure 11, there is no fluctuation observed. In the LTE network, eNodeB use a link adaption procedure to improve spectral efficiency. This mechanism relies on the Adaptive Modulation and Coding (AMC) scheme based on a CQI report [23]. The fluctuation in the power received by the UE is due to this mechanism. Since there is interference from Wi-Fi, eNodeB changes the transmission power based on the CQI report received.

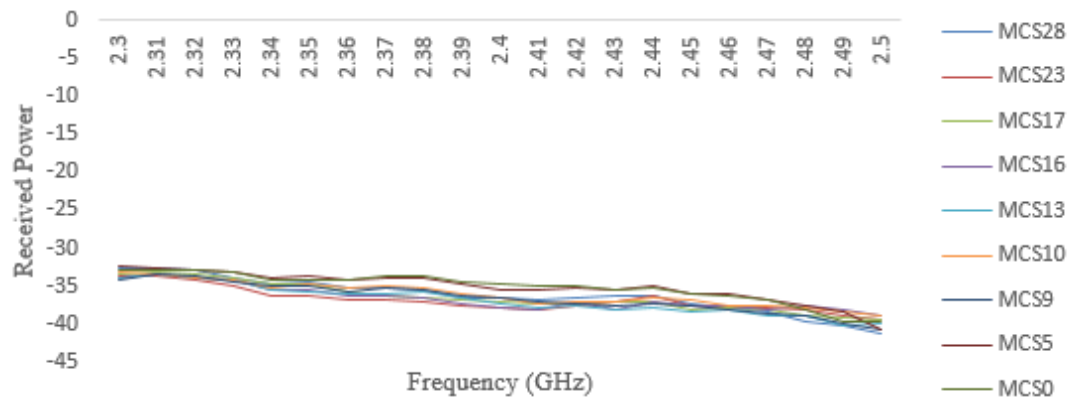

Figure 10. Power received by UE for FDD operation mode without Wi-Fi signal

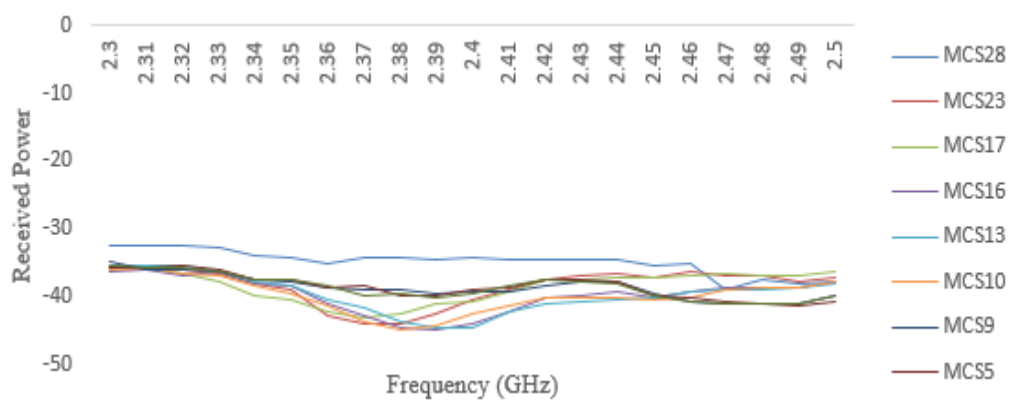

Figure 11. Power received by UE for TDD operation mode without Wi-Fi signal

\section{CONCLUSION}

This paper evaluated the LTE data transmission with the coexistence of the $2.4 \mathrm{GHz}$ Wi-Fi signal. The experimental results showed that there is a performance degradation in the LTE system with the coexistence of a Wi-Fi signal. The results also showed that the degradation on the LTE throughput increase with higher MCS values. The video throughput of FDD operation mode is higher than the TDD operation mode, but the TDD operation mode has experienced less power fluctuation compared to FDD since TDD has less effect from the Wi-Fi signal. Since the LTE network uses the CQI mechanism, the performance of the LTE network is affected by the interference from the Wi-Fi signal in the heterogeneous wireless network.

\section{ACKNOWLEDGEMENTS}

This work was supported in part by TM Research and Development under Grant MMUE/170013.

\section{REFERENCES}

[1] G. Piro, L. A. Grieco, G. Boggia, F. Capozzi, and P. Camarda, "Simulating LTE Cellular Systems: An OpenSource Framework," IEEE Trans. Veh. Technol., vol. 60, no. 2, pp. 498-513, Feb. 2011.

[2] M. F. L. Abdullah and A. Z. Yonis, "Performance of LTE Release 8 and Release 10 in wireless communications," in Proceedings Title: 2012 International Conference on Cyber Security, Cyber Warfare and Digital Forensic (CyberSec), 2012, pp. 236-241.

[3] E. H. Salman, N. K. Noordin, S. J. Hashim, F. Hashim, and C. K. Ng, "An overview of spectrum sensing techniques for cognitive LTE and LTE-A radio systems," Telecommun. Syst., vol. 65, no. 2, pp. 215-228, Jun. 2017. 
[4] Z. Shen, A. Papasakellariou, J. Montojo, D. Gerstenberger, and F. Xu, "Overview of 3GPP LTE-advanced carrier aggregation for 4G wireless communications," IEEE Commun. Mag., vol. 50, no. 2, pp. 122-130, Feb. 2012.

[5] S. Kanchi, S. Sandilya, D. Bhosale, A. Pitkar, and M. Gondhalekar, "Overview of LTE-A technology," in 2013 IEEE Global High Tech Congress on Electronics, 2013, pp. 195-200.

[6] A. Ghosh, R. Ratasuk, B. Mondal, N. Mangalvedhe, and T. Thomas, "LTE-advanced: next-generation wireless broadband technology [Invited Paper," IEEE Wirel. Commun., vol. 17, no. 3, pp. 10-22, Jun. 2010.

[7] J. Góra, "QoS-aware resource management for LTE-Advanced relay-enhanced network," EURASIP J. Wirel. Commun. Netw., vol. 2014, no. 1, p. 178, Dec. 2014.

[8] L. Zeng and Q. Cui, "A novel cross-carrier scheduling method in carrier aggregation," in 2013 International Conference on Computational Problem-Solving (ICCP), 2013, pp. 312-315.

[9] G. Yuan, X. Zhang, W. Wang, and Y. Yang, "Carrier aggregation for LTE-advanced mobile communication systems," IEEE Commun. Mag., vol. 48, no. 2, pp. 88-93, Feb. 2010.

[10] T.-T. Tran, Y. Shin, and O.-S. Shin, "Overview of enabling technologies for 3GPP LTE-advanced," EURASIP J. Wirel. Commun. Netw., vol. 2012, no. 1, p. 54, Dec. 2012.

[11] Nokia Solutions and Networks, "Enhance mobile networks to deliver 1000 times more capacity by 2020," 2014.

[12] Xinsheng Zhao, Zhiyi Guo, and Qiang Guo, "A cognitive based spectrum sharing scheme for LTE advanced systems," in International Congress on Ultra Modern Telecommunications and Control Systems, 2010, pp. 965-969.

[13] S. Arunthavanathan, S. Kandeepan, and R. J. Evans, "Spectrum sensing and detection of incumbent-UEs in secondary-LTE based aerial-terrestrial networks for disaster recovery," in 2013 IEEE 18th International Workshop on Computer Aided Modeling and Design of Communication Links and Networks (CAMAD), 2013, pp. 201-206.

[14] N. Alyaoui, A. Kachouri, and M. Samet, "The fourth generation 3GPP LTE identification for cognitive radio," in ICM 2011 Proceeding, 2011, pp. 1-5.

[15] C. Tsai and T. Lee, "Uplink performance enhancement for LTE licensed-assisted access to unlicensed spectrum," 2018.

[16] Y.-Y. Liu and S.-J. Yoo, "Dynamic resource allocation using reinforcement learning for LTE-U and WiFi in the unlicensed spectrum," 2017 Ninth Int. Conf. Ubiquitous Futur. Networks, pp. 471-475, 2017.

[17] S. Zinno, G. Di Stasi, S. Avallone, and G. Ventre, "On a fair coexistence of LTE and Wi-Fi in the unlicensed spectrum: A Survey," Comput. Commun., vol. 115, no. September 2017, pp. 35-50, 2018.

[18] Haitao Wu, Yong Peng, Keping Long, Shiduan Cheng, and Jian Ma, "Performance of reliable transport protocol over IEEE 802.11 wireless LAN: analysis and enhancement," in Proceedings.Twenty-First Annual Joint Conference of the IEEE Computer and Communications Societies, vol. 2, pp. 599-607.

[19] N. Rastegardoost and B. Jabbari, "WiFi White Spaces for Opportunistic LTE-U," in GLOBECOM 2017 - 2017 IEEE Global Communications Conference, 2017, pp. 1-6.

[20] E. Dahlman, S. Parkvall, and J. Skold, "4G LTE-advanced pro and the road to 5G. Elsevier, 2016.

[21] S. Compliance, "LabVIEW Communications 802.11 Application Framework White Paper," 2015. [Online]. Available: http://www.ni.com/white-paper/52524/en/.

[22] C. Cox, "An Introduction to LTE. 2012.

[23] S. Avallone, N. Pasquino, S. Zinno, and D. Casillo, "Experimental characterization of LTE adaptive modulation and coding scheme under actual operating conditions," 2017 IEEE Int. Work. Meas. Netw., pp. 1-6, 2017.

[24] L. T. E. A. Framework and G. S. Guide, "LTE Application Framework 2.0.1 Getting Started Guide,"

[25] D. López-Pérez, Á. Ladányi, A. Jüttner, H. Rivano, and J. Zhang, "Optimization method for the joint allocation of modulation schemes, coding rates, resource blocks and power in self-organizing LTE networks," Proc. - IEEE INFOCOM, pp. 111-115, 2011.

\section{BIOGRAPHIES OF AUTHORS}

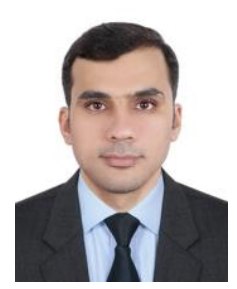

Yaqoob J. I. A. Yghoubi received B.E degree in Electronics from Multimedia University, Malaysia in March 2017. Currently a M.Eng.Sc student at Multimedia University, Malaysia. His research interests focus on the media access control, scheduling algorithms and quality of service in the OFDMA-based wireless network.

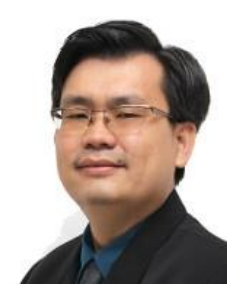

Wai-Leong Pang received his Ph.D. degree in 2013. He is a Professional Engineer with Practising Certificate with Board of Engineers Malaysia and a corporate member with The Institution of Engineers Malaysia. His research interests include wireless communications, networking, VLSI, digital design and renewable energy. Since 2004, he has been with Multimedia University, Malaysia. Currently, he is the Programme Coordinator of the B.Eng. (Hons) Electronics under Faculty of Engineering, Multimedia University, Malaysia. 


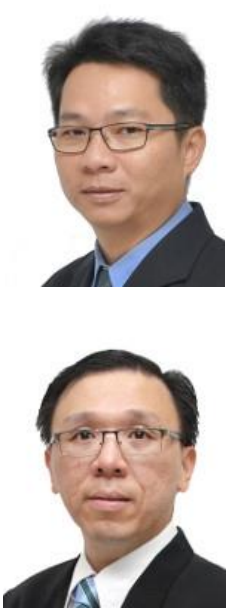

Sew-Kin Wong received his B.Eng degree in Electrical and Electronic Engineering in 1995. He then obtained his M.Eng.Sc degree and Ph.D degree in 2003 and 2011 respectively. His research interests are RF transceiver and RFIC design for the next generation wireless systems and EMI/EMC compliances and shielding techniques. He is currently a member of the Faculty of Engineering, Multimedia University, Malaysia.

Kah-Yoong Chan obtained his Ph.D. in Electrical Engineering from Jacobs University Bremen in Germany in year 2008. He is a Professional Engineer with Practising Certificate (PEPC) with BEM, a senior member with IEEE, and a corporate member with IEM. Ir. Dr. Chan research areas are in the micro and nanoelectronic device fabrications and characterizations. Currently, he is an Associate Professor with Faculty of Engineering in Multimedia University, and serving as a chaiperson for a Research Center under Faculty of Engineering of MMU - Centre for Advanced Devices and Systems (CADS). 